\title{
Übergangsmetall- und Lanthanoid-Komplexe von 1,4,7,10-Tetraallyl- und 1,4,7,10-Tetra-3-butenyl-1,4,7,10-tetraazacyclododecan
}

Transition Metal and Lanthanide Complexes of 1,4,7,10-Tetraallyl- and 1,4,7,10-Tetra-3-butenyl1,4,7,10-tetraazacyclododecane

Herbert Schumann, Katharina Kuse, Markus Hummert und Joachim Pickardt

Institut für Chemie, Technische Universität Berlin, Straße des 17. Juni 135, D-10623 Berlin, Germany

Reprint requests to Prof. Dr. H. Schumann. Fax: +49 3031422168.

E-mail: Schumann@tu-berlin.de

Z. Naturforsch. 2009, 64b, 93 -100; received November 12, 2008

Professor Otto J. Scherer zum 75. Geburtstag gewidmet

1,4,7,10-Tetraazacyclododecane (1) reacts with allylbromide and 3-butenylbromide giving 1,4,7,10-tetraallyl-1,4,7,10-tetraazacyclododecane (TAC) (2a) and 1,4,7,10-tetra-3-butenyl-1,4,7,10tetraazacyclododecane (TBC) (2b), respectively. Compounds $\mathbf{2 a}$ and $\mathbf{2 b}$ react with $\mathrm{FeCl}_{2}, \mathrm{CoCl}_{2}$, $\mathrm{RhCl}_{3}, \mathrm{NiCl}_{2}, \mathrm{CuBr}_{2}$, and $\mathrm{TmCl}_{3}$ forming the complexes [ $\left.\mathrm{FeCl}(\mathrm{TAC})\right] \mathrm{Cl}(\mathbf{3 a}),[\mathrm{FeCl}(\mathrm{TBC})] \mathrm{Cl}(\mathbf{3 b})$, $[\mathrm{CoCl}(\mathrm{TAC})] \mathrm{Cl}(\mathbf{4}),[\mathrm{RhCl}(\mathrm{TAC})] \mathrm{Cl}_{2}(\mathbf{5}),[\mathrm{NiCl}(\mathrm{TAC})] \mathrm{Cl}(\mathbf{6 a}),[\mathrm{NiCl}(\mathrm{TBC})] \mathrm{Cl}(\mathbf{6 b}),[\mathrm{CuBr}(\mathrm{TAC})] \mathrm{Br}$ (7), $[\mathrm{TmCl}(\mathrm{TAC})] \mathrm{Cl}_{2}(\mathbf{8 a})$, and $[\mathrm{TmCl}(\mathrm{TBC})] \mathrm{Cl}_{2}(\mathbf{8 b})$. The reaction of $\mathbf{6 a}$ with an excess of $\mathrm{CuCl}$ affords a coordination polymer in which $[\mathrm{NiCl}(\mathrm{TAC})]^{+}$cations are connected by $\left[\mathrm{Cu}_{6} \mathrm{Cl}_{8}\right]^{2-}$ anions (9). The ${ }^{1} \mathrm{H}$ and ${ }^{13} \mathrm{C}$ NMR spectra of $\mathbf{2 a}, \mathbf{2 b}, \mathbf{5}$, and $\mathbf{6 a}$, as well as the single crystal X-ray structures of $2 \mathbf{a} \cdot 3 \mathbf{H C l}, 6 \mathbf{a}, 6 \mathbf{b}, 7$, and 9 are reported and discussed.

Key words: Tetraazacyclododecane, Cobalt, Copper, Iron, Nickel, Rhodium, Thulium, Complexes

\section{Einleitung}

Cyclische Amine wie 1,4,7-Triazacyclononan [1] oder 1,4,7,10-Tetraazacyclododecan [2] und vor allem deren an den Stickstoffatomen mit Carboxylatgruppen substituierte Derivate, wie 1,4,7,10-Tetraazacyclododecan-1,4,7,10-tetraessigsäure (DOTA) [3], bilden als mehrzähnige Liganden mit fast allen Metallen in wässriger Lösung thermodynamisch und kinetisch äußerst stabile Komplexe. So bildet beispielsweise DOTA in wässriger Lösung mit Gadolinium(III)Kationen das komplexe Anion $\left[\mathrm{Gd}(\mathrm{DOTA})\left(\mathrm{H}_{2} \mathrm{O}\right)\right]^{-}$, in dem das Gadoliniumatom durch Koordination von vier Stickstoffatomen, vier Carboxylatanionen und einem Wassermolekül wie in einem Käfig gefangen gehalten und gleichzeitig die für Gd(III) ideale Koordinationszahl 9 erreicht wird [4]. Der schnelle Austausch der Wassermoleküle zwischen den einzelnen Komplexanionen ermöglicht die Verwendung dieser Substanz im medizinischen Bereich als Kontrastmittel in der Magnetresonanz-Tomographie (MRT) [5]. Vielfach dienen die auf cyclischen Ligandensystemen basieren- den Metallkomplexe auch als Modelle für das Studium der Protein-Metall-Bindungsstellen in biologischen Systemen [1-3], als selektive Komplexbildner für Metallionen [4-8] wie etwa bei der therapeutischen Behandlung von Metallvergiftungen $[9,10]$, als Radiopharmaka in der Nuklearmedizin [11,12], als Leuchtsonden oder Schalter [13] oder zur Spaltung von RNA [14]. Makrocyclische Komplexe mit einem substituierten Cyclensystem weisen darüberhinaus anti-Tumor- oder anti-HIV-Aktivitäten auf [15-18].

Besonderes Interesse gilt seit einiger Zeit der Entwicklung bifunktioneller Liganden, die einerseits in der Lage sind, durch harte Lewis-basische Donoratome elektropositive Übergangsmetalle zu chelatisieren und andererseits über weiche Lewis-basische funktionelle Gruppen elektronenreiche Übergangsmetalle wie Rhodium oder Iridium zu binden [19]. So haben Baker et al. auf das Metathesepotential von Komplexen alkenylfunktionalisierter 1,4,7-Triazacyclononane hingewiesen [20].

An dieser Stelle berichten wir über die im Rahmen unserer Arbeiten zur Synthese funktionalisier- 
ter Tetraazacyclododecane durchgeführte Darstellung und Charakterisierung zweier tetraalkenylsubstituierter Derivate und einiger ihrer Metallkomplexe.

\section{Synthese und Eigenschaften}

Synthese der Liganden $\left(\mathrm{CH}_{2} \mathrm{CH}_{2} \mathrm{NCH}_{2} \mathrm{CH}=\mathrm{CH}_{2}\right)_{4}$ (2a) und $\left(\mathrm{CH}_{2} \mathrm{CH}_{2} \mathrm{NCH}_{2} \mathrm{CH}_{2} \mathrm{CH}=\mathrm{CH}_{2}\right)_{4}(2 \boldsymbol{b})$

Die Synthese von 1,4,7,10-Tetraallyl- (2a, TAC) und 1,4,7,10-Tetra-3-butenyl-1,4,7,10-tetraazacyclododecan (2b, TBC) gelingt durch direkte Alkylierung von 1,4,7,10-Tetraazacyclododecan (1) mit Allyl- bzw. 3-Butenylchlorid in siedendem Acetonitril (Schema 1). Nach säulenchromatographischer Trennung von mono-, di- und trialkylierten Nebenprodukten werden die Liganden in 80 bis $90 \%$ Ausbeute als farblose Öle gewonnen. Die Elementaranalysen sowie die IR-, ${ }^{1} \mathrm{H}-\mathrm{NMR}$ - und ${ }^{13} \mathrm{C}-\mathrm{NMR}-$ Spektren von 2a und $\mathbf{2 b}$ und das Massenspektrum von $\mathbf{2 b}$ bestätigen die Zusammensetzung und Konstitution der beiden Verbindungen. Während das ${ }^{1} \mathrm{H}-\mathrm{NMR}$-Signal für die Protonen des Ringsystems von $\mathbf{2 a}$ und $\mathbf{2 b}$ nur geringfügig gegenüber dem entsprechenden Signal des unsubstituierten Cyclens (2.69 ppm) verschoben ist [21], zeigt das ${ }^{13} \mathrm{C}$-NMR-Signal für die Ringkohlenstoffatome gegenüber dem Signal des unsubstituierten Cyclens jeweils eine Verschiebung um 3 ppm zu tieferem Feld.

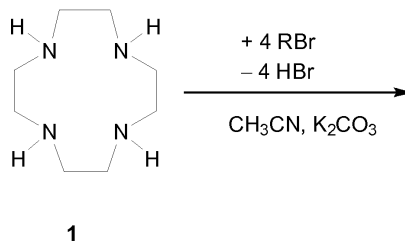

Schema 1.

Synthese der Komplexe [FeCl(TAC)]Cl (3a), [FeCl $(\mathrm{TBC})] \mathrm{Cl}(\mathbf{3 b}),[\mathrm{CoCl}(\mathrm{TAC})] \mathrm{Cl}(4),[\mathrm{RhCl}(\mathrm{TAC})] \mathrm{Cl}_{2}$ (5), [NiCl(TAC)]Cl (6a), [NiCl(TBC)]Cl (6b), [CuBr (TAC) $] \mathrm{Br}(\mathbf{7}),[\mathrm{TmCl}(\mathrm{TAC})] \mathrm{Cl}_{2}$ (8a) und [TmCl(TBC)] $\mathrm{Cl}_{2}(\mathbf{8 b})$

Die Liganden 1,4,7,10-Tetraallyl-1,4,7,10-tetraazacyclododecan (2a, TAC) und 1,4,7,10-Tetra-3butenyl-1,4,7,10-tetraazacyclododecan (2b, TBC) reagieren mit $\mathrm{FeCl}_{2}, \mathrm{CoCl}_{2}, \mathrm{RhCl}_{3}\left(\mathrm{H}_{2} \mathrm{O}\right)_{6}, \mathrm{NiCl}_{2}$ und $\mathrm{TmCl}_{3}\left(\mathrm{H}_{2} \mathrm{O}\right)_{6}$ in THF bzw. mit $\mathrm{CuBr}_{2}$ in Ethanol bei erhöhten Temperaturen im Verlauf mehrerer Stunden
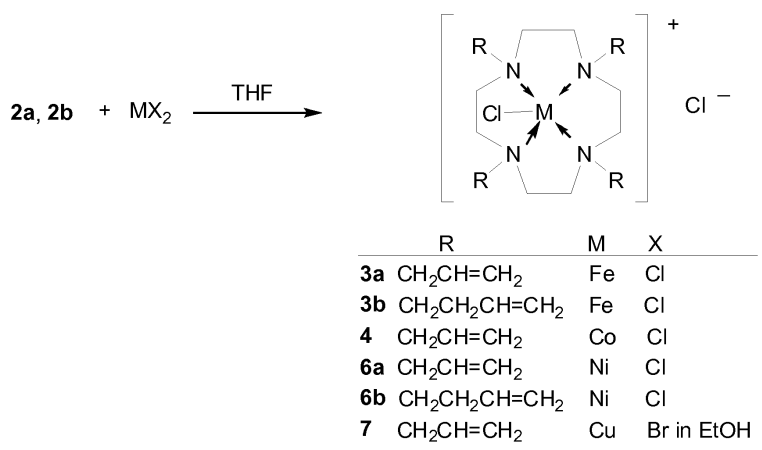

$$
\text { 2a, 2b }+\mathrm{MCl}_{3} \stackrel{\mathrm{THF}}{\longrightarrow}
$$
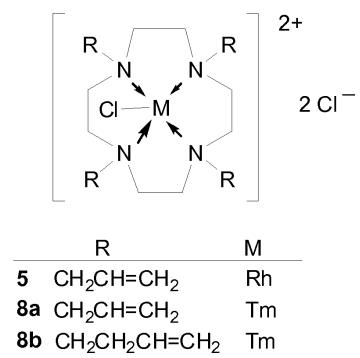

Schema 2.

unter Bildung von $[\mathrm{FeCl}(\mathrm{TAC})] \mathrm{Cl}(\mathbf{3 a}),[\mathrm{FeCl}(\mathrm{TBC})]$ $\mathrm{Cl}$ (3b), $[\mathrm{CoCl}(\mathrm{TAC})] \mathrm{Cl}$ (4), $[\mathrm{RhCl}(\mathrm{TAC})] \mathrm{Cl}_{2}$ (5), $[\mathrm{NiCl}(\mathrm{TAC})] \mathrm{Cl}(\mathbf{6 a}),[\mathrm{NiCl}(\mathrm{TBC})] \mathrm{Cl}(\mathbf{6 b}), \quad[\mathrm{CuBr}$ (TAC) $] \mathrm{Br}$ (7), $[\mathrm{TmCl}(\mathrm{TAC})] \mathrm{Cl}_{2}$ (8a) und $[\mathrm{TmCl}$ (TBC) $\mathrm{Cl}_{2}(\mathbf{8 b})$. Die als farblose $(\mathbf{3 a}, \mathbf{3 b}, \mathbf{4})$, hellbraune (5), hellgrüne (6a), dunkelgrüne (6b), blaue (7) oder beigefarbene Feststoffe $(\mathbf{8 a}, \mathbf{8 b})$ ausfallenden Verbindungen werden in Ausbeuten zwischen 40 und $80 \%$ isoliert (Schema 2). Die Röntgenstrukturanalysen zeigen, dass bei $\mathbf{6 a}[\mathrm{NiCl}(\mathrm{TAC})]^{+}$, bei $\mathbf{6 b}[\mathrm{NiCl}(\mathrm{TBC})]^{+}$ und bei $7[\mathrm{CuBr}(\mathrm{TAC})]^{+}$als Kationen und $\mathrm{Cl}^{-}$bzw. $\mathrm{Br}^{-}$als Anionen vorliegen. Wir nehmen deshalb an, dass die röntgenographisch nicht untersuchten Komplexe eine analoge Molekülstruktur besitzen.

Die von den Komplexen in $\mathrm{D}_{2} \mathrm{O}$ und $\left[\mathrm{D}_{8}\right] \mathrm{DMSO}$ $(\mathbf{5}, \mathbf{6 a})$ aufgenommenen ${ }^{1} \mathrm{H}$ - und ${ }^{13} \mathrm{C}$-NMR-Spektren zeigen Signale und Signalgruppen, die sich durch Vergleich mit den Spektren des freien Cyclens 2a eindeutig zuordnen lassen.

In den Absorptionsspektren der beiden Thuliumkomplexe 8a und $\mathbf{8 b}$, aufgenommen in wässriger Lösung $\left(10^{-2}\right.$ bzw. $\left.10^{-3} \mathrm{M}\right)$, treten Absorptionsbanden bei $\lambda_{\text {max }}=310 \mathrm{~nm}(\mathbf{8 a})$ bzw. 255 und $334 \mathrm{~nm}(\mathbf{8 b})$ auf. Nach Anregung mit UV-Strahlung $\left(\lambda_{\mathrm{Ex} 1}=240 \mathrm{~nm}\right.$ und $\lambda_{\mathrm{Ex} 2}=310 \mathrm{~nm}$ bei $\mathbf{8 a}$ und $\lambda_{\mathrm{Ex}}=433 \mathrm{~nm}$ bei $\mathbf{8 b}$ ) ist ein Fluoreszieren der beiden Verbindungen entsprechend $\lambda_{\mathrm{F} 11}=252 \mathrm{~nm}(\mathrm{I}=29.2)$ und $\lambda_{\mathrm{Fl} 2}=350 \mathrm{~nm}(\mathrm{I}=$ 
40.8) für 8a und $\lambda_{\mathrm{Fl}}=486 \mathrm{~nm}(\mathrm{I}=19.0)$ für $\mathbf{8 b} \mathrm{zu}$ beobachten.

Die Ergebnisse cyclovoltammetrischer Messungen an den Eisenkomplexen $\mathbf{3 a}$ und $\mathbf{3 b}$ sowie dem Rhodiumkomplex 5 verweisen auf $\mathrm{Fe}^{2+} / \mathrm{Fe}^{3+}$ bzw. $\mathrm{Rh}^{3+} / \mathrm{Rh}^{4+}$ Oxidationsprozesse, während im Falle des Cobaltkomplexes $\mathbf{4}$ eine irreversible Reduktion $\mathrm{Co}^{2+} / \mathrm{Co}^{1+}$ angezeigt wird. Über diese elektrochemischen Untersuchungen wird an anderer Stelle gesondert und ausführlich berichtet.

\section{Bildung von $\left\{[\mathrm{NiCl}(\mathrm{TAC})]_{2}\left[\mathrm{Cu}_{6} \mathrm{Cl}_{8}\right]\right\}_{n}$ (9)}

Die bei Raumtemperatur unter Stickstoff in absolutem Acetonitril durchgeführte eintägige Umsetzung von $[\mathrm{NiCl}(\mathrm{TAC})] \mathrm{Cl}$ (6a) mit überschüssigem $\mathrm{CuCl}$ liefert nach entsprechender Aufarbeitung des Reaktionsgemischs dunkelgrüne, rautenförmige Kristalle, die sich erst oberhalb $220{ }^{\circ} \mathrm{C}$ zersetzen. Die Röntgenstrukturanalyse von einem dieser Kristalle zeigte, dass sich ein Koordinationspolymer gebildet hat, in dem [ $\mathrm{NiCl}(\mathrm{TAC})]$ Einheiten über einen $\left[\mathrm{Cu}_{6} \mathrm{Cl}_{8}\right]$-Cluster miteinander vernetzt sind (Schema 3). Die Kristalle zeigen im ${ }^{1} \mathrm{H}-\mathrm{NMR}$-Spektrum drei breite Multipletts bei 8.84, 11.67 und 13.81 ppm.

\section{Röntgenstrukturanalysen}

1,4,7,10-Tetraallyl-1,4,7,10-tetraazacyclododekan (2a) wird aus Ethanol/Wasser als Hydrochlorid

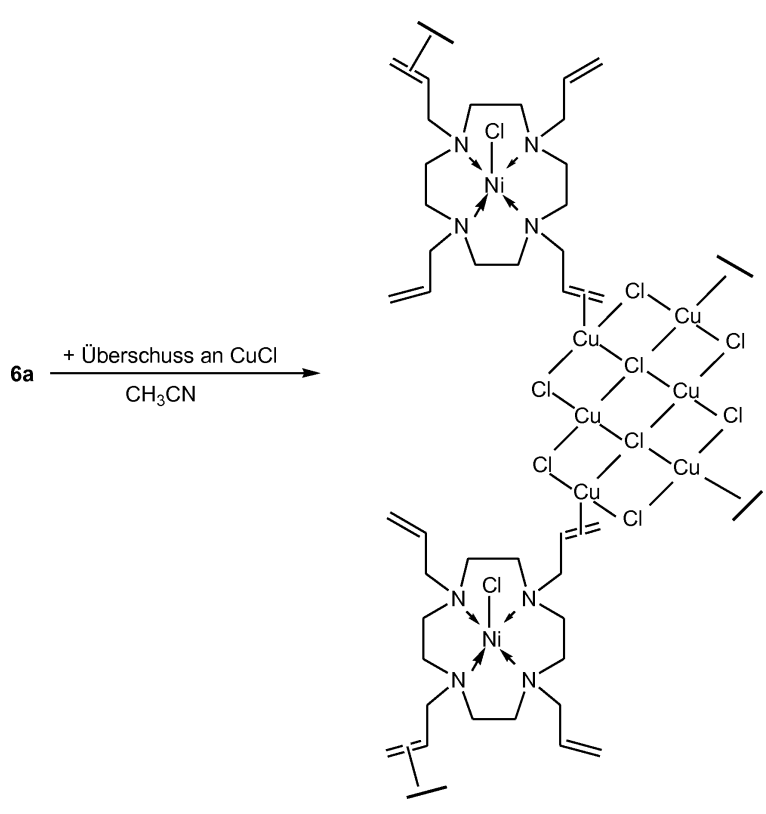

Schema 3.

9

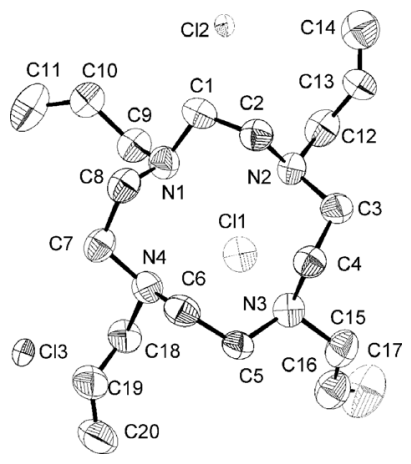

Abb. 1. Molekülstruktur von 2a-3HCl (Auslenkungsellipsoide mit $35 \%$ Aufenthaltswahrscheinlichkeit). Bindungslängen und -winkel liegen im Erwartungsbereich.

2a.3HCl in Form farbloser Kristalle erhalten. Sie kristallisieren monoklin in der zentrosymmetrischen Raumgruppe $P 2_{1} / c$ mit 4 Formeleinheiten pro Elementarzelle. Die C-N-Einfachbindungslängen liegen im Mittel bei $1.488 \AA$. Die terminalen $\mathrm{C}=\mathrm{C}$ Bindungen der Allylsubstituenten zeigen eine Länge von $1.26 \AA$ und sind damit etwas kürzer als der durchschnittliche Wert von $1.299 \AA$ für C=C-Doppelbindungen. Die terminalen C-Atome der Allylketten sind geringfügig fehlgeordnet, was sich in großen Auslenkungsparametern äußert. $\mathrm{Cl}(1)$, das Chloratom eines der drei $\mathrm{HCl}$ Moleküle, liegt etwa $3 \AA$ über dem Ringzentrum mit dem kürzesten Abstand zu N(3) (3.17 ̊) (Abb. 1).

$[\mathrm{NiCl}(\mathrm{TAC})] \mathrm{Cl}(\mathbf{6 a})$ kristallisiert aus Acetonitril in Form dunkelgrüner Kristalle in der monokli-

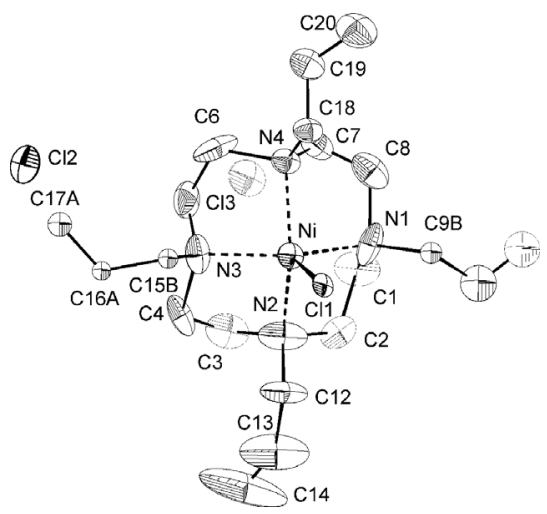

Abb. 2. Molekülstruktur von 6a (Auslenkungsellipsoide mit $35 \%$ Aufenthaltswahrscheinlichkeit). Ausgewählte Bindungslängen $[\AA]$ und -winkel $\left[^{\circ}\right]$ : $\mathrm{Ni}-\mathrm{N}(1)$ 2.064(13), $\mathrm{Ni}-\mathrm{N}(2)$ 2.072(13), Ni-N(3) 2.053(11), Ni-N(4) 2.086(12), $\mathrm{Ni}-\mathrm{Cl}(1) \quad 2.365(7) ; \mathrm{N}(1)-\mathrm{Ni}-\mathrm{N}(2) \quad 84.9(7), \mathrm{N}(1)-\mathrm{Ni}-\mathrm{N}(3)$ 147.9(4), N(1)-Ni-N(4) 85.6(6), N(2)-Ni-N(3) 86.1(6), $\mathrm{N}(2)-\mathrm{Ni}-\mathrm{N}(4)$ 148.2(4), N(3)-Ni-N(4) 86.1(5), N(1)-Ni$\mathrm{Cl}(1) \quad 104.2(3), \quad \mathrm{N}(2)-\mathrm{Ni}-\mathrm{Cl}(1)$ 105.0(3), N(3)-Ni-Cl(1) 107.9(3), N(4)-Ni-Cl(1) 106.7(3). 
nen zentrosymmetrischen Raumgruppe $C 2 / c$. Die Allyl-Seitenketten sind zum Teil stark fehlgeordnet. Als einzig verlässlicher Wert für die $\mathrm{C}=\mathrm{C}$ Doppelbindungslänge kann der Abstand von $1.314 \AA$ zwischen C(19) und C(20) angesehen werden. Das Nickelatom liegt $0.563 \AA$ über der von den vier Stickstoffatomen gebildeten Ebene und befindet sich innerhalb einer quadratischen Pyramide, gebildet aus den vier Stickstoffatomen an den Ecken der Basisfläche und dem Chloratom $\mathrm{Cl}(1)$ an der Spitze. Der mittlere Ni-N Abstand beträgt $2.069 \AA$ und der Abstand $\mathrm{Ni}-\mathrm{Cl}(1) 2.365(7) \AA$ A. Der Abstand zwischen dem Nickelatom und $\mathrm{Cl}(2)$ ist mit 4.809(1) $\AA$ zu lang, um im Sinne einer koordinativen Bindung interpretiert werden zu können (Abb. 2).

Für die Röntgenstrukturanalyse geeignete Kristalle von $[\mathrm{NiCl}(\mathrm{TBC})] \mathrm{Cl}(\mathbf{6 b})$ erhält man durch Kristallisation aus Methanol. Sie kristallisieren in der monoklinen Raumgruppe $P 2_{1} / n$. Analog zur Molekülstruktur von 6a ist das Nickelatom im Molekül 6b fünffach koordiniert und befindet sich innerhalb einer quadratischen Pyramide, in der die Ecken der Basisfläche von den vier N-Atomen eingenommen werden, und die Spitze von einem Chloratom besetzt ist. Der Abstand des Nickelatoms von der Grundfläche der Pyramide beträgt $0.575 \AA$ und besitzt damit nahezu den gleichen Wert wie der entsprechende Abstand in Verbindung $6 \mathbf{a}$. Der Abstand $\mathrm{Ni}-\mathrm{Cl}(1)$ ist dagegen etwas kleiner wie

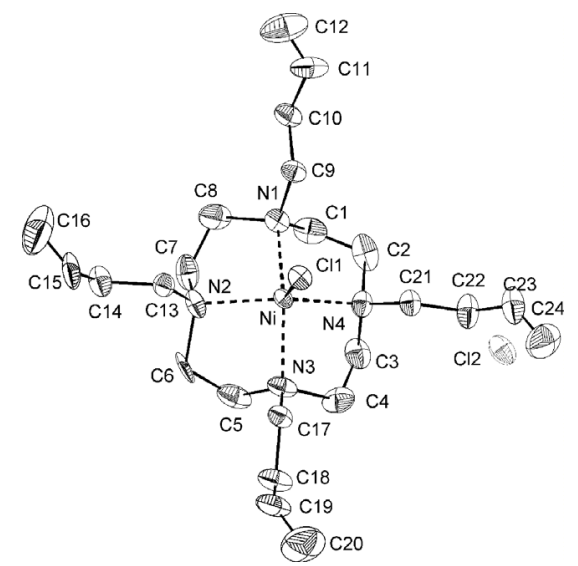

Abb. 3. Molekülstruktur von 6b (Auslenkungsellipsoide mit $35 \%$ Aufenthaltswahrscheinlichkeit). Ausgewählte Bindungslängen $[\AA]$ und -winkel $\left[{ }^{\circ}\right]$ : $\mathrm{Ni}-\mathrm{N}(1)$ 2.094(6), $\mathrm{Ni}-\mathrm{N}(2)$ 2.096(6), Ni-N(3) 2.107(6), Ni-N(4) 2.090(6), $\mathrm{Ni}-\mathrm{Cl}(1)$ 2.272(2); N(1)-Ni-N(2) 86.7(3), N(1)-Ni-N(3) 148.1(2), N(1)-Ni-N(4) 85.2(3), N(2)-Ni-N(3) 85.5(3), $\mathrm{N}(2)-\mathrm{Ni}-\mathrm{N}(4)$ 148.2(2), N(3)-Ni-N(4) 85.3(3), N(1)-Ni$\mathrm{Cl}(1) \quad 105.3(2), \quad \mathrm{N}(2)-\mathrm{Ni}-\mathrm{Cl}(1) \quad 105.8(2), \mathrm{N}(3)-\mathrm{Ni}-\mathrm{Cl}(1)$ 106.6(2), N(4)-Ni-Cl(1) 106.0(2).

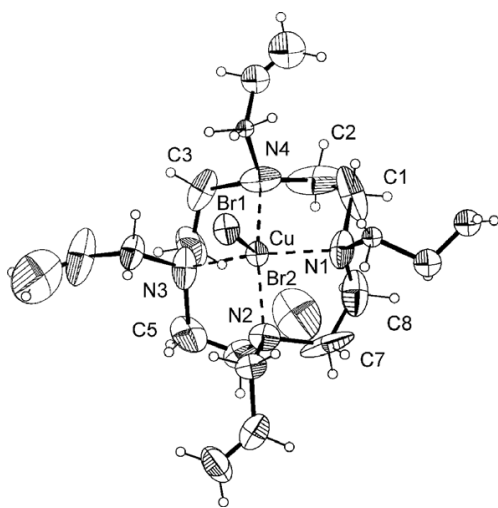

Abb. 4. Molekülstruktur von 7 (Auslenkungsellipsoide mit $35 \%$ Aufenthaltswahrscheinlichkeit). Ausgewählte Bindungslängen $[\AA]$ und -winkel $\left[{ }^{\circ}\right]$ : $\mathrm{N}(1)-\mathrm{Cu}(1) 2.060(16)$, $\mathrm{N}(2)-\mathrm{Cu}(1) 2.031(16), \mathrm{N}(3)-\mathrm{Cu}(1)$ 2.049(16), N(4)-Cu(1) 2.103(16), $\mathrm{Br}(1)-\mathrm{Cu}(1)$ 2.530(3); $\mathrm{N}(1)-\mathrm{Cu}(1)-\mathrm{N}(2)$ 87.9(8), $\mathrm{N}(1)-\mathrm{Cu}(1)-\mathrm{N}(3) \quad 147.9(7), \quad \mathrm{N}(1)-\mathrm{Cu}(1)-\mathrm{N}(4) \quad 83.9(8)$, $\mathrm{N}(2)-\mathrm{Cu}(1)-\mathrm{N}(3) \quad 84.8(8), \quad \mathrm{N}(2)-\mathrm{Cu}(1)-\mathrm{N}(4) \quad 148.9(6)$, $\mathrm{N}(3)-\mathrm{Cu}(1)-\mathrm{N}(4) \quad 86.4(9), \quad \mathrm{N}(1)-\mathrm{Cu}(1)-\mathrm{Br}(1) \quad$ 108.4(5), $\mathrm{N}(2)-\mathrm{Cu}(1)-\mathrm{Br}(1)$ 105.1(4), $\mathrm{N}(3)-\mathrm{Cu}(1)-\mathrm{Br}(1)$ 103.7(5), $\mathrm{N}(4)-\mathrm{Cu}(1)-\operatorname{Br}(1)$ 105.9(4).

in 6a und beträgt 2.272(2) $\AA$. Auch in dieser Verbindung weisen die Kohlenstoffatome der Seitenketten mit zunehmendem Abstand vom jeweiligen Stickstoffatom größer werdende Auslenkungskoeffizienten auf, so dass eine Diskussion der C-C-Bindungslängen der Butenylsubstituenten nicht sinnvoll erscheint (Abb. 3).

$[\mathrm{CuBr}(\mathrm{TAC})] \mathrm{Br}$ (7) kristallisiert aus einem Ethanol/Wasser-Gemisch in Form blauer monokliner Kristalle der Raumgruppe $C 2 / c$. In jeder Elementarzelle sind acht Formeleinheiten enthalten. Auch hier sind die Allylketten zum Teil stark fehlgeordnet, und damit ist eine Diskussion der darin vorliegenden Bindungslängen wenig sinnvoll. In Abb. 4 sind diese Ketten deshalb idealisiert dargestellt. Wie in $\mathbf{6 a}$ und $\mathbf{6 b}$ liegt das Kupferatom in Komplex 7 fünffach koordiniert vor und ist quadratisch pyramidal von den vier die Basis der Pyramide bildenden Stickstoffatomen des TAC-Liganden und dem Bromatom [Br(1)] an der Pyramidenspitze umgeben. Der Abstand des Kupferatoms von der Basisfläche betrtägt $0.560 \AA$. Der mittlere $\mathrm{Cu}-\mathrm{N}-\mathrm{Abstand}$ hat einen Wert von $2.061 \AA$, und der Abstand $\mathrm{Cu}-\mathrm{Br}(1)$ entspricht 2.530(3) $\AA$. $\operatorname{Br}(2)$ ist vom Komplexkation zu weit entfernt, um mit ihm in diskrete Wechselwirkung treten zu können.

$\left\{[\mathrm{NiCl}(\mathrm{TAC})]_{2}\left[\mathrm{Cu}_{6} \mathrm{Cl}_{8}\right]\right\}_{n}(\mathbf{9})$ kristallisiert aus Acetonitril in Form dunkelgrüner rautenförmiger Kristalle der triklinen zentrosymmetrischen Raumgrup- 


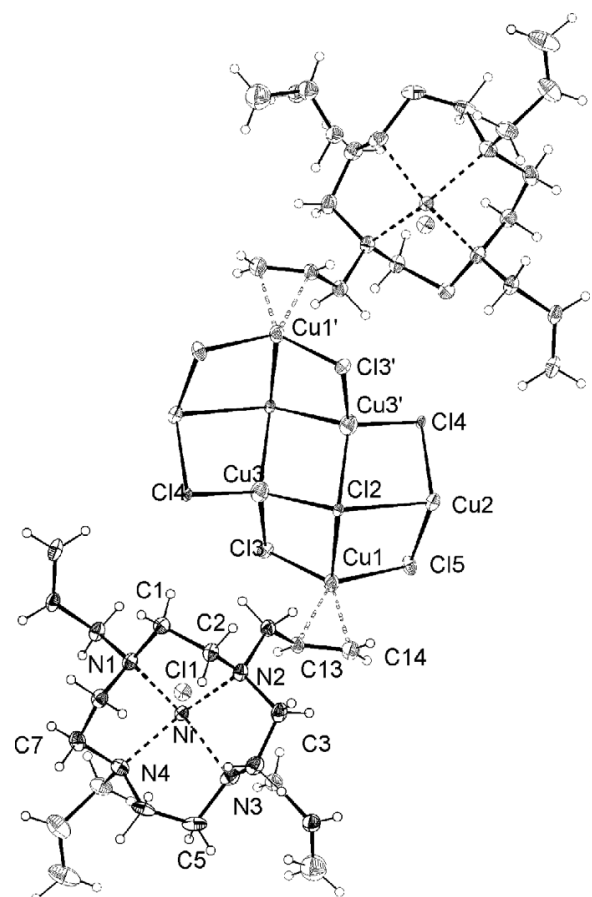

Abb. 5. Ausschnitt aus der Koordinationspolymerkette von 9 (Auslenkungsellipsoide mit $35 \%$ Aufenthaltswahrscheinlichkeit). Ausgewählte Bindungslängen [尺] und -winkel [ $\left.{ }^{\circ}\right]$ : Ni-N(1) 2.091(5), Ni-N(2) 2.120(5), Ni-N(3) 2.078(5), Ni-N(4) 2.122(6), Ni-Cl(1) 2.279(2), Cu(1)-Cl(2) 2.605(2), $\mathrm{Cu}(1)-\mathrm{Cl}(3) \quad 2.323(2), \mathrm{Cu}(1)-\mathrm{Cl}(5) \quad 2.340(2)$, $\mathrm{Cu}(2)-\mathrm{Cl}(4)$ 2.310(2), $\mathrm{Cu}(2)-\mathrm{Cl}(5)$ 2.287(2), $\mathrm{Cu}(3)-\mathrm{Cl}(2)$ 2.484(2), $\mathrm{Cu}(3)-\mathrm{Cl}(3) \quad 2.341(2), \mathrm{Cu}(3)-\mathrm{Cl}(4) \quad 2.351(2)$, $\mathrm{Cu}(1)-\mathrm{C}(13)$ 2.106(6), $\mathrm{Cu}(1)-\mathrm{C}(14)$ 2.071(7); N(1)-Ni$\mathrm{N}(2) \quad 85.8(2), \quad \mathrm{N}(1)-\mathrm{Ni}-\mathrm{N}(3) \quad 147.3(2), \quad \mathrm{N}(1)-\mathrm{Ni}-\mathrm{N}(4)$ 85.4(2), N(2)-Ni-N(3) 85.3(2), N(2)-Ni-N(4) 147.6(2), $\mathrm{N}(3)-\mathrm{Ni}-\mathrm{N}(4)$ 85.4(2), N(1)-Ni-Cl(1) 105.8(2), N(2)-Ni$\mathrm{Cl}(1) \quad 110.1(2), \quad \mathrm{N}(3)-\mathrm{Ni}-\mathrm{Cl}(1)$ 106.8(2), N(4)-Ni-Cl(1) 102.3(2), $\mathrm{Cl}(2)-\mathrm{Cu}(1)-\mathrm{Cl}(3)$ 100.68(5), $\mathrm{Cl}(2)-\mathrm{Cu}(1)-\mathrm{Cl}(5)$ 97.59(5), $\mathrm{Cl}(3)-\mathrm{Cu}(1)-\mathrm{Cl}(5)$ 107.06(6), $\mathrm{Cl}(2)-\mathrm{Cu}(2)-\mathrm{Cl}(4)$ 99.26(6), $\mathrm{Cl}(2)-\mathrm{Cu}(2)-\mathrm{Cl}(5)$ 92.62(6), $\mathrm{Cl}(4)-\mathrm{Cu}(2)-\mathrm{Cl}(5)$ 110.11(6), $\mathrm{Cl}(2)-\mathrm{Cu}(3)-\mathrm{Cl}(3) 103.80(6), \mathrm{Cl}(2)-\mathrm{Cu}(3)-\mathrm{Cl}(4)$ 108.37(6), C(13)-Cu(1)-C(4) 38.8(3).

pe $P \overline{1}$ mit zwei Formeleinheiten pro Elementarzelle. Pro Formeleinheit ist ein fehlgeordnetes Acetonitrilmolekül im Kristall enthalten. Im Gegensatz zu den bisher beschriebenen Komplexen ist 9 polymer aufgebaut. Es besteht aus $[\mathrm{NiCl}(\mathrm{TAC})]^{+}$-Einheiten, die über zwei ihrer vier Allylgruppen einwertige Kupferionen koordinieren. Diese sind ihrerseits Bestandteil von $\left[\mathrm{Cu}_{6} \mathrm{Cl}_{8}\right]^{2-}$-Clustern. Auf diese Weise werden Bänder gebildet, die sich ungefähr in Richtung der $a$-Achse durch den Kristall ziehen (Abb. 5). Der geringste Abstand zwischen zwei Bändern besteht zwischen den Atomen $\mathrm{Cl}(3)$ und Ni und beträgt $4.895 \AA$. Die Geo- metrie der $[\mathrm{NiCl}(\mathrm{TAC})]^{+}$-Kationen entspricht der tetragonal pyramidalen Geometrie der bereits beschriebenen Ni-Komplexe 6a und $\mathbf{6 b}$. Der mittlere Ni-NAbstand ist jedoch etwas größer und beträgt 2.103 A. Damit einher geht auch ein größerer Abstand des Nickelatoms zu der von den vier Stickstoffatomen gebildeten Basisebene der Pyramide von $0.590 \AA$ A. Die $\mathrm{Ni}(1)-\mathrm{Cl}(1)$ Bindung ist mit 2.279(1) $\AA$ etwas kürzer als im monomeren Komplex 6a. Die $\left[\mathrm{Cu}_{6} \mathrm{Cl}_{8}\right]^{2-}$ Cluster zeigen sesselartige Konformation mit zentrosymmetrischer Atomanordnung. $\mathrm{Cu}(3)$ ist ausschließlich von vier Chloratomen umgeben, während $\mathrm{Cu}(1)$ und $\mathrm{Cu}(2)$ jeweils neben den Bindungen zu drei Chloratomen eine koordinative Bindung $\mathrm{zu}$ einer terminalen $\mathrm{C}=\mathrm{C}$-Doppelbindung unterschiedlicher $[\mathrm{NiCl}(\mathrm{TAC})]^{+}$ Einheiten aufweisen. Die Koordination des Olefins an $\mathrm{Cu}(1)$ erfolgt side-on. Die Abstände von $\mathrm{Cu}(1) \mathrm{zu}$ den Kohlenstoffatomen C(13) und C(14) der AllylDoppelbindung betragen 2.106(6) bzw. 2.071(7) $\AA$. Ähnliche Werte wurden für den $\mathrm{Cu}^{+}$-Ethen-Komplex $\mathrm{Cu}\left[\mathrm{HB}\left(3,5-\mathrm{Me}_{2} \mathrm{pz}\right)_{3}\right]\left(\mathrm{C}_{2} \mathrm{H}_{4}\right)$ mit 2.023(5)/2.004(6) $\AA$ gefunden [22]. Die $\mathrm{Cu}-\mathrm{Cl}-\mathrm{Abstände}$ liegen zwischen 2.29 und $2.84 \AA$.

\section{Experimenteller Teil}

Alle Arbeiten wurden mit sorgfältig getrockneten und entgasten Lösemitteln in Schlenk-Kolben in einer Atmosphäre von gereinigtem Stickstoff durchgeführt. $\mathrm{CoCl}_{2}$, $\mathrm{CuCl}, \mathrm{CuBr}_{2}, \mathrm{FeCl}_{2}, \mathrm{NiCl}_{2}$ und $\mathrm{TmCl}_{3}\left(\mathrm{H}_{2} \mathrm{O}\right)_{6}$ wurden von Aldrich bezogen, $\mathrm{RhCl}_{3}\left(\mathrm{H}_{2} \mathrm{O}\right)_{3}$ wurde von der Degussa AG zur Verfügung gestellt. $\left(\mathrm{HNCH}_{2} \mathrm{CH}_{2}\right)_{4}$ (Cyclen) wurde aus $\mathrm{HN}\left(\mathrm{CH}_{2} \mathrm{CH}_{2} \mathrm{NH}_{2}\right)_{2}$ nach Literaturvorschriften [2326] hergestellt. ${ }^{1} \mathrm{H}$ - und ${ }^{13} \mathrm{C}-\mathrm{NMR}$ : Bruker ARX 200 und ARX 400 (200/400 bzw. 50.32/100.64 MHz), $\delta$ in ppm gegen TMS. IR: Magna System 750, Nicolet, He-Ne-Laser in $\mathrm{KBr}$. UV/VIS: Perkin Elmer Lambda 20, Software: UV WINLAB V.4, $\lambda=190-900 \mathrm{~nm}$. Fluorimetrie: Shimadzu RF-1502. Cyclovoltammetrie: $\mathrm{CH}_{3} \mathrm{CN} / \mathrm{Bu}_{4} \mathrm{NBF}_{4}$ (Leitelektrolyt $c=0.1 \mathrm{~mol} \mathrm{~L}^{-1}$ ), Sweeprate: $200 \mathrm{mV} / \mathrm{s} ; \mathrm{Ag} / \mathrm{AgCl} / \mathrm{KCl}$ gesättigt (Bezugselektrode), Pt-Arbeitselektrode $(2 \mathrm{~mm})$, Pt-Stab (Hilfselektrode). CH-Analysen: Perkin-Elmer 240C CHNS/O Analyzer.

\section{$\left(\mathrm{CH}_{2} \mathrm{CH}_{2} \mathrm{NCH}_{2} \mathrm{CH}=\mathrm{CH}_{2}\right)_{4}(2 \mathrm{a})$}

$\mathrm{Zu}$ einer Lösung von $1.21 \mathrm{~g}(7.02 \mathrm{mmol})\left(\mathrm{HNCH}_{2} \mathrm{CH}_{2}\right)_{4}$

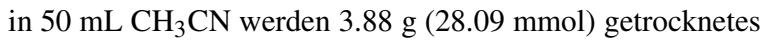
$\mathrm{K}_{2} \mathrm{CO}_{3}$ gegeben und das Gemisch auf $60^{\circ} \mathrm{C}$ erwärmt. $\mathrm{Zu}$ diesem Gemisch wird im Verlauf von 80 min eine Lösung von frisch destilliertem $\mathrm{CH}_{2}=\mathrm{CHCH}_{2} \mathrm{Br}(3.74 \mathrm{~g}, 30.9 \mathrm{mmol})$ in $20 \mathrm{~mL} \mathrm{CH}{ }_{3} \mathrm{CN}$ getropft, die Mischung $3 \mathrm{~h}$ auf $80{ }^{\circ} \mathrm{C}$ erhitzt und anschließend $14 \mathrm{~h}$ bei $40^{\circ} \mathrm{C}$ gerührt. Nach Abkühlen auf 
Raumtemperatur wird das Lösemittel im Vacuum abgezogen, der Rückstand in $\mathrm{CH}_{2} \mathrm{Cl}_{2}$ aufgenommen und die filtrierte organische Phase mehrmals mit Wasser ausgeschüttelt. Nach dem Trocknen der organischen Phase über $\mathrm{Na}_{2} \mathrm{SO}_{4}$, Filtration und Abtrennung des Lösemittels im Vacuum verbleibt 2a als farbloses Öl, das säulenchromatographisch über Kieselgel $\left(\mathrm{CH}_{2} \mathrm{Cl}_{2} / \mathrm{MeOH}: 3: 1\right)$ gereinigt wird. Ausbeute: $1.9 \mathrm{~g}$ (81\%). - IR (KBr): $v=1638(\mathrm{C}=\mathrm{C}) \mathrm{cm}^{-1} .-{ }^{1} \mathrm{H}-\mathrm{NMR}$ $\left(200 \mathrm{MHz}, \mathrm{CDCl}_{3}\right): \delta=2.64\left(\mathrm{~s}, 16 \mathrm{H}, \mathrm{N}\left(\mathrm{CH}_{2}\right)_{2} \mathrm{~N}\right), 3.08(\mathrm{~d}$, $\left.J=6.84 \mathrm{~Hz}, 8 \mathrm{H}, \mathrm{NCH}_{2} \mathrm{CH}=\mathrm{CH}_{2}\right), 4.92\left(\mathrm{~d}, 4 \mathrm{H}, \mathrm{CH}=\mathrm{CH}_{2}\right)$, $5.08\left(\mathrm{~d}, 4 \mathrm{H}, \mathrm{CH}=\mathrm{CH}_{2}\right), 5.40-5.68\left(\mathrm{~m}, 4 \mathrm{H}, \mathrm{CH}=\mathrm{CH}_{2}\right)$. ${ }^{13} \mathrm{C}$-NMR $\left(200 \mathrm{MHz}, \mathrm{CDCl}_{3}\right): \delta=49.15\left(\mathrm{NCH}_{2} \mathrm{CH}_{2} \mathrm{~N}\right)$, $56.04\left(\mathrm{CH}_{2} \mathrm{C}_{2} \mathrm{H}_{3}\right), 120.71\left(\mathrm{CH}=\mathrm{CH}_{2}\right), 130.55\left(\mathrm{CH}=\mathrm{CH}_{2}\right)$. $\mathrm{C}_{20} \mathrm{H}_{36} \mathrm{~N}_{4}$ (332.53): ber. C 72.24, H 10.91, N 16.85; gef. C 72.54, H 11.02, N 16.64.

\section{$\left(\mathrm{CH}_{2} \mathrm{CH}_{2} \mathrm{NCH}_{2} \mathrm{CH}_{2} \mathrm{CH}=\mathrm{CH}_{2}\right)_{4}(2 \boldsymbol{b})$}

Analog zur Synthese von 2a werden $1.00 \mathrm{~g}(5.81 \mathrm{mmol})$ $\left(\mathrm{HNCH}_{2} \mathrm{CH}_{2}\right)_{4}$ in $50 \mathrm{~mL} \mathrm{CH} \mathrm{CN}_{3}$ gelöst und in Gegenwart von $3.32 \mathrm{~g}(24.0 \mathrm{mmol}) \mathrm{K}_{2} \mathrm{CO}_{3}$ mit $3.21 \mathrm{~g}(23.8 \mathrm{mmol})$ $\mathrm{CH}_{2}=\mathrm{CHCH}_{2} \mathrm{CH}_{2} \mathrm{Br}$ in $15 \mathrm{~mL} \mathrm{CH} \mathrm{CH}_{3} \mathrm{CN}$ zur Reaktion gebracht und das Reaktionsgemisch $14 \mathrm{~h}$ unter Rückfluss erhitzt. Nach entsprechender Aufarbeitung des Reaktionsgemischs erhält man eine sich in 2 Phasen trennende ölige Flüssigkeit. Die farblose Phase, bestehend aus reinem $\mathbf{2 b}$, wird abdekantiert, und die zweite Phase, bestehend aus verunreinigtem Produkt, wird säulenchromatographisch gereinigt $\left(\mathrm{CH}_{2} \mathrm{Cl}_{2} / \mathrm{MeOH}: 1: 2\right)$ und mit der ersten Phase vereinigt. Ausbeute: $1.97 \mathrm{~g}(88 \%)$. - IR (KBr): $v=1640$ $(\mathrm{C}=\mathrm{C}) \mathrm{cm}^{-1} .-{ }^{1} \mathrm{H}-\mathrm{NMR}\left(200 \mathrm{MHz}, \mathrm{CDCl}_{3}\right): \delta=2.14-$ 2.29 (q, $\left.8 \mathrm{H}, \mathrm{CH}_{2} \mathrm{C}_{2} \mathrm{H}_{3}\right), 2.42-2.54\left(\mathrm{~m}, 8 \mathrm{H}, \mathrm{CH}_{2} \mathrm{C}_{3} \mathrm{H}_{5}\right.$ ), $2.65\left(\mathrm{~s}, 16 \mathrm{H}, \mathrm{N}\left(\mathrm{CH}_{2}\right)_{2} \mathrm{~N}\right), 4.91-5.13\left(\mathrm{dq}, 8 \mathrm{H}, \mathrm{CH}=\mathrm{CH}_{2}\right)$, $5.69-5.95\left(\mathrm{dt}, 4 \mathrm{H}, \mathrm{CH}=\mathrm{CH}_{2}\right) .-{ }^{13} \mathrm{C}-\mathrm{NMR}(200 \mathrm{MHz}$, $\left.\mathrm{CDCl}_{3}\right): \delta=31.68\left(\mathrm{CH}_{2} \mathrm{C}_{2} \mathrm{H}_{3}\right), 52.15\left(\mathrm{NCH}_{2} \mathrm{CH}_{2} \mathrm{~N}\right), 55.37$ $\left(\mathrm{CH}_{2} \mathrm{C}_{3} \mathrm{H}_{5}\right), 115.27\left(\mathrm{CH}=\mathrm{CH}_{2}\right), 137.17\left(\mathrm{CH}=\mathrm{CH}_{2}\right) .-\mathrm{MS}$ $\left(\mathrm{EI}, 162{ }^{\circ} \mathrm{C}\right): m / z(\%)=193(78)[\mathrm{M} / 2-\mathrm{H}]^{+}, 167(10)$ $\left[\mathrm{N}\left(\mathrm{C}_{4} \mathrm{H}_{7}\right)\left(\mathrm{CH}_{2}\right)_{2}+\mathrm{H}\right]^{+}, 98(68)\left[\mathrm{N}\left(\mathrm{C}_{4} \mathrm{H}_{7}\right)\left(\mathrm{CH}_{2}\right)_{2}-\mathrm{H}\right]^{+}$,

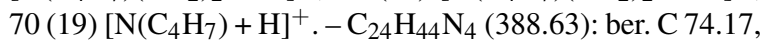
H 11.41, N 14.42; gef. C 74.28, H 11.56, N 14.53.

\section{$\left[\mathrm{FeCl}\left(\mathrm{CH}_{2} \mathrm{CH}_{2} \mathrm{NCH}_{2} \mathrm{CH}=\mathrm{CH}_{2}\right)_{4}\right] \mathrm{Cl}(3 \mathrm{a})$}

$\mathrm{Zu} 0.11 \mathrm{~g}(0.33 \mathrm{mmol}) \mathbf{2 a}$, gelöst in $10 \mathrm{~mL}$ THF, werden $0.04 \mathrm{~g}(0.33 \mathrm{mmol}) \mathrm{FeCl}_{2}$ gegeben und das Reaktionsgemisch unter Rühren 12 h zum Sieden erhitzt. Nach dem Abkühlen wird das als farbloser Niederschlag ausgefallene 3a unter Luftausschluss abfiltriert, mit THF gewaschen und im Vakuum getrocknet. Ausbeute: $0.08 \mathrm{~g}(53 \%)$.Schmp.: $210{ }^{\circ} \mathrm{C}$. $-\mathrm{C}_{20} \mathrm{H}_{36} \mathrm{Cl}_{2} \mathrm{FeN}_{4}$ (459.28): ber. C 52.30, H 7.90, N 12.20; gef. C 53.05, H 8.12, N 12.47.

\section{$\left[\mathrm{FeCl}\left(\mathrm{CH}_{2} \mathrm{CH}_{2} \mathrm{NCH}_{2} \mathrm{CH}_{2} \mathrm{CH}=\mathrm{CH}_{2}\right)_{4}\right] \mathrm{Cl}(3 \boldsymbol{b})$}

In Analogie zur Darstellung von 3a wird farbloses 3b durch Umsetzung von $1.01 \mathrm{~g}(2.60 \mathrm{mmol}) 2 \mathrm{~b}$ mit $0.36 \mathrm{~g}$
( $2.84 \mathrm{mmol}) \mathrm{FeCl}_{2}$ in $50 \mathrm{~mL}$ THF erhalten. Ausbeute: $0.61 \mathrm{~g}$ (46\%). $-\mathrm{C}_{24} \mathrm{H}_{44} \mathrm{Cl}_{2} \mathrm{FeN}_{4}$ (515.38): ber. C 55.93, $\mathrm{H}$ 8.61, N 10.87; gef. C 56.24, H 8.86, N 11.12.

\section{$\left[\mathrm{CoCl}\left(\mathrm{CH}_{2} \mathrm{CH}_{2} \mathrm{NCH}_{2} \mathrm{CH}=\mathrm{CH}_{2}\right)_{4}\right] \mathrm{Cl}(4)$}

In Analogie zur Darstellung von 3a wird farbloses 4 durch Umsetzung von $0.13 \mathrm{~g}(0.39 \mathrm{mmol}) \mathbf{2 a}$ mit $0.05 \mathrm{~g}$ ( $0.39 \mathrm{mmol}) \mathrm{CoCl}_{2}$ in $10 \mathrm{~mL}$ THF erhalten. Ausbeute: $0.08 \mathrm{~g}$ (43\%). - Schmp.: $216^{\circ} \mathrm{C}$. $-\mathrm{C}_{20} \mathrm{H}_{36} \mathrm{Cl}_{2} \mathrm{CoN}_{4}$ (462.37): ber. C 51.95, H 7.85, N 12.12; gef. C 50.15, H 7.48, N 11.65.

\section{$\left[\mathrm{RhCl}\left(\mathrm{CH}_{2} \mathrm{CH}_{2} \mathrm{NCH}_{2} \mathrm{CH}=\mathrm{CH}_{2}\right)_{4}\right] \mathrm{Cl}_{2}(5)$}

In Analogie zur Darstellung von 3a wird hellbraunes 5 durch Umsetzung von $0.27 \mathrm{~g}(0.81 \mathrm{mmol}) \mathbf{2 a}$ mit $0.21 \mathrm{~g}$ (0.81 mmol) $\mathrm{RhCl}_{3}\left(\mathrm{H}_{2} \mathrm{O}\right)_{3}$ in $20 \mathrm{~mL}$ THF erhalten. Ausbeute: $0.26 \mathrm{~g}$ (59\%). - Schmp.: > $300{ }^{\circ} \mathrm{C}$ (Zers.). ${ }^{1} \mathrm{H}-\mathrm{NMR}\left(200 \mathrm{MHz},\left[\mathrm{D}_{8}\right] \mathrm{DMSO}\right): \delta=2.64-2.90(\mathrm{~m}$, $4 \mathrm{H}, \mathrm{N}\left(\mathrm{CH}_{2} \mathrm{CH}_{2} \mathrm{~N}\right), 2.92-3.15\left(\mathrm{~m}, 8 \mathrm{H}, \mathrm{NCH}_{2} \mathrm{CH}_{2} \mathrm{~N}\right)$, $3.16-3.41\left(\mathrm{~m}, 4 \mathrm{H}, \mathrm{NCH}_{2} \mathrm{CH}_{2} \mathrm{~N}\right), 3.48-3.69(\mathrm{~m}, 8 \mathrm{H}$, $\left.\mathrm{CH}_{2} \mathrm{C}_{2} \mathrm{H}_{3}\right), 5.21-5.74\left(\mathrm{~m}, 8 \mathrm{H}, \mathrm{CH}=\mathrm{CH}_{2}\right), 5.86-6.26(\mathrm{~m}$, $\left.4 \mathrm{H}, \mathrm{CH}=\mathrm{CH}_{2}\right) .-\mathrm{C}_{20} \mathrm{H}_{36} \mathrm{Cl}_{3} \mathrm{~N}_{4} \mathrm{Rh}$ (541.79): ber. $\mathrm{C} 44.34$, H 6.70, N 10.34; gef. C 44.20, H 6.89, N 10.52 .

\section{$\left[\mathrm{NiCl}\left(\mathrm{CH}_{2} \mathrm{CH}_{2} \mathrm{NCH}_{2} \mathrm{CH}=\mathrm{CH}_{2}\right)_{4}\right] \mathrm{Cl}(\boldsymbol{6} \boldsymbol{a})$}

In Analogie zur Darstellung von 3a wird hellgrünes 6a durch Umsetzung von $0.85 \mathrm{~g}$ (2.56 mmol) 2a mit $0.35 \mathrm{~g}$ (2.70 mmol) $\mathrm{NiCl}_{2}$ in $80 \mathrm{~mL}$ THF erhalten. Ausbeute: $0.99 \mathrm{~g}$ (81\%). - Schmp.: $>220{ }^{\circ} \mathrm{C}$ (Zers.). $-{ }^{1} \mathrm{H}-\mathrm{NMR}$ (200 MHz, $\left.\mathrm{D}_{2} \mathrm{O}\right): \delta=2.89-3.17\left(\mathrm{~m}, 16 \mathrm{H}, \mathrm{N}\left(\mathrm{CH}_{2} \mathrm{CH}_{2} \mathrm{~N}\right), 3.34-\right.$ $3.53\left(\mathrm{~m}, 8 \mathrm{H}, \mathrm{CH}_{2} \mathrm{C}_{2} \mathrm{H}_{3}\right), 5.26-5.49\left(\mathrm{~m}, 8 \mathrm{H}, \mathrm{CH}=\mathrm{CH}_{2}\right)$, 5.67-5.94 (m, $\left.4 \mathrm{H}, \mathrm{CH}=\mathrm{CH}_{2}\right) .-{ }^{1} \mathrm{H}-\mathrm{NMR}$; (200 MHz, [D $\mathrm{D}_{8}$ ]DMSO): $\delta=2.72-3.18\left(\mathrm{~m}, 16 \mathrm{H}, \mathrm{NCH}_{2} \mathrm{CH}_{2} \mathrm{~N}\right), 3.20-$ $3.60\left(\mathrm{~m}, 8 \mathrm{H}, \mathrm{CH}_{2} \mathrm{C}_{2} \mathrm{H}_{3}\right), 5.04-5.47\left(\mathrm{~m}, 8 \mathrm{H}, \mathrm{CH}=\mathrm{CH}_{2}\right)$, $5.74-6.20\left(\mathrm{~m}, 4 \mathrm{H}, \mathrm{CH}=\mathrm{CH}_{2}\right) .-{ }^{13} \mathrm{C}-\mathrm{NMR}(200 \mathrm{MHz}$, $\left.\mathrm{D}_{2} \mathrm{O}\right): \delta=47.72\left(\mathrm{NCH}_{2} \mathrm{CH}_{2} \mathrm{~N}\right), 55.36\left(\mathrm{CH}_{2} \mathrm{C}_{2} \mathrm{H}_{3}\right), 124.69$ $\left(\mathrm{CH}=\mathrm{CH}_{2}\right), 127.55\left(\mathrm{CH}=\mathrm{CH}_{2}\right) .-{ }^{13} \mathrm{C}-\mathrm{NMR}(200 \mathrm{MHz}$, [D $\mathrm{D}_{8}$ ]DMSO): $\delta=45.07\left(\mathrm{NCH}_{2} \mathrm{CH}_{2} \mathrm{~N}\right), 52.06\left(\mathrm{CH}_{2} \mathrm{C}_{2} \mathrm{H}_{3}\right)$, $119.71\left(\mathrm{CH}=\mathrm{CH}_{2}\right), 126.54\left(\mathrm{CH}=\mathrm{CH}_{2}\right) .-\mathrm{C}_{20} \mathrm{H}_{36} \mathrm{Cl}_{2} \mathrm{~N}_{4} \mathrm{Ni}$ (562.13): ber. C 51.98, H 7.85, N 12.12; gef. C 52.13, H 7.95, N 12.24 .

\section{$\left[\mathrm{NiCl}\left(\mathrm{CH}_{2} \mathrm{CH}_{2} \mathrm{NCH}_{2} \mathrm{CH}_{2} \mathrm{CH}=\mathrm{CH}_{2}\right)_{4}\right] \mathrm{Cl}(\boldsymbol{6} \boldsymbol{b})$}

In Analogie zur Darstellung von 3a wird dunkelgrünes $\mathbf{6 b}$ durch Umsetzung von $0.25 \mathrm{~g}$ (0.64 mmol) 2b mit $0.09 \mathrm{~g}$ (0.69 mmol) $\mathrm{NiCl}_{2}$ in $70 \mathrm{~mL}$ THF erhalten. Ausbeute: $0.28 \mathrm{~g}$ (82\%). - Schmp.: $>220{ }^{\circ} \mathrm{C}$ (Zers.). $-\mathrm{C}_{24} \mathrm{H}_{44} \mathrm{Cl}_{2} \mathrm{~N}_{4} \mathrm{Ni}$ (518.23): ber. C 55.62, H 8.56, N 10.81; gef. C 55.77, H 8.66, N 10.99 .

\section{$\left[\mathrm{CuBr}\left(\mathrm{CH}_{2} \mathrm{CH}_{2} \mathrm{NCH}_{2} \mathrm{CH}=\mathrm{CH}_{2}\right)_{4}\right] \mathrm{Br}$ (7)}

In Analogie zur Darstellung von 3a wird blaues 7 durch Umsetzung von $0.05 \mathrm{~g}(0.17 \mathrm{mmol}) 2 \mathrm{2a}$ mit $0.04 \mathrm{~g}$ 
Tabelle 1. Kristall- und Messparamater zu den Kristallstrukturanalysen.

\begin{tabular}{|c|c|c|c|c|c|}
\hline Verbindung & $2 \mathbf{a} \cdot 3 \mathrm{HCl}$ & $\mathbf{6 a}$ & $\mathbf{6 b}$ & 7 & 9 \\
\hline Formeleinheit & $\mathrm{C}_{20} \mathrm{H}_{36} \mathrm{ClN}_{4} \times 3 \mathrm{HCl}$ & $\mathrm{C}_{20} \mathrm{H}_{36} \mathrm{Cl}_{2} \mathrm{~N}_{4} \mathrm{Ni}$ & $\begin{array}{l}\mathrm{C}_{24} \mathrm{H}_{44} \mathrm{Cl}_{2} \mathrm{~N}_{4} \\
\mathrm{Ni} \times \mathrm{CH}_{3} \mathrm{OH}\end{array}$ & $\mathrm{C}_{20} \mathrm{H}_{36} \mathrm{Br}_{2} \mathrm{CuN}_{4}$ & $\begin{array}{l}\mathrm{C}_{20} \mathrm{H}_{36} \mathrm{Cl}_{5} \\
\mathrm{Cu}_{3} \mathrm{~N}_{4} \mathrm{Ni} \times \mathrm{CH}_{3} \mathrm{CN}\end{array}$ \\
\hline$M_{w}\left[\mathrm{~g} \mathrm{~mol}^{-1}\right]$ & 441.90 & 462.30 & 550.28 & 555.88 & 800.16 \\
\hline Kristallgröße $\left[\mathrm{mm}^{3}\right]$ & $0.54 \times 0.43 \times 0.33$ & $0.52 \times 0.46 \times 0.32$ & $0.75 \times 0.71 \times 0.43$ & $0.68 \times 0.53 \times 0.43$ & $0.38 \times 0.38 \times 0.36$ \\
\hline Kristallsystem & monoklin & monoklin & monoklin & monoklin & triklin \\
\hline Raumgruppe & $P 2_{1} / c$ & $C 2 / c$ & $P 2_{1} / n$ & $C 2 / c$ & $P \overline{1}$ \\
\hline$Z$ (Formeleinheiten) & 4 & 8 & 4 & 8 & 2 \\
\hline$a[\AA]$ & $14.680(5)$ & $35.008(3)$ & $15.2977(11)$ & $35.4290(17)$ & $10.1562(3)$ \\
\hline$b[\AA]$ & $13.339(5)$ & $10.5009(9)$ & $8.1699(6)$ & $10.4718(5)$ & $11.0131(4)$ \\
\hline$c[\AA]$ & $12.525(5)$ & 14.7668 & $24.6673(16)$ & $15.0516(8)$ & $15.7114(5)$ \\
\hline$\alpha\left[^{\circ}\right]$ & 90 & 90 & 90 & 90 & $103.5060(10)$ \\
\hline$\beta\left[^{\circ}\right]$ & $96.338(5)$ & $111.771(5)$ & 101.812 & $111.501(2)$ & $95.2160(10)$ \\
\hline$\gamma\left[{ }^{\circ}\right]$ & 90 & 90 & 90 & 90 & $114.4200(10)$ \\
\hline$V\left[\AA^{3}\right]$ & $2437.6(16)$ & $5041.3(7)$ & $3017.7(4)$ & $5195.6(4)$ & $1520.60(9)$ \\
\hline$D_{\mathrm{c}}\left[\mathrm{g} \mathrm{cm}^{-3}\right]$ & 1.204 & 1.374 & 1.211 & 1.503 & 1.737 \\
\hline Absorpt.koeff. [mm $\left.{ }^{-1}\right]$ & 0.388 & 1.108 & 0.842 & 3.938 & 3.060 \\
\hline Absorpt.korr. $T_{\max } / T_{\min }$ & $0.8453 / 0.5988$ & $0.8684 / 0.4915$ & $0.9879 / 0.3615$ & $0.94562 / 0.63329$ & $0.46162 / 0.63329$ \\
\hline$F(000)[\mathrm{e}]$ & 952 & 22003 & 1184 & 2392 & 810 \\
\hline Messbereich $\theta_{\min } / \theta_{\max }\left[^{\circ}\right]$ & $2.07 / 25.00$ & $1.25 / 25.00$ & $2.62 / 25.00$ & $1.24 / 25.00$ & $1.36 / 25.00$ \\
\hline \multirow[t]{3}{*}{ Indexbereich } & $-16 \leq h \leq 17$ & $-30 \leq h \leq 41$ & $-16 \leq h \leq 18$ & $-42 \leq h \leq 42$ & $-10 \leq h \leq 12$ \\
\hline & $-15 \leq k \leq 15$ & $-12 \leq k \leq 12$ & $-9 \leq k \leq 9$ & $-12 \leq k \leq 12$ & $-13 \leq k \leq 12$ \\
\hline & $-13 \leq l \leq 14$ & $-17 \leq l \leq 13$ & $-27 \leq l \leq 29$ & $-16 \leq l \leq 17$ & $-18 \leq l \leq 18$ \\
\hline Reflexe, gemessen & $1493 \overline{8}$ & 15539 & $1841 \overline{5}$ & $1602 \overline{3}$ & 9558 \\
\hline Reflexe unabhängig & 4281 & 4456 & 5323 & 4566 & 5325 \\
\hline$R_{\text {int }}$ & 0.1266 & 0.2198 & 0.1699 & 0.2125 & 0.0577 \\
\hline Daten/Restraints/Parameter & $4281 / 0 / 245$ & $4456 / 0 / 263$ & $5323 / 0 / 291$ & $4566 / 6 / 259$ & $5325 / 0 / 383$ \\
\hline Goodness-of-fit $\left(F^{2}\right)$ & 1.583 & 1.176 & 1.016 & 1.042 & 1.057 \\
\hline$R_{1} / w R_{2}[I \geq 2 \sigma(I)]$ & $0.1566 / 0.3936$ & $0.1186 / 0.3273$ & $0.0818 / 0.1880$ & $0.1161 / 0.3133$ & $0.0604 / 0.1607$ \\
\hline$R_{1} / w R_{2}$ (alle Daten) & $0.2009 / 0.4274$ & $0.2052 / 0.3948$ & $0.1508 / 0.2210$ & $0.2405 / 0.3837$ & $0.0727 / 0.1721$ \\
\hline Restelektronend. $\left[\mathrm{e} \AA^{-3}\right]$ & $3.44 /-0.77$ & $1.33 /-1.07$ & $0.76 /-0.49$ & $1.80 /-1.89$ & $1.82 /-1.50$ \\
\hline
\end{tabular}

(0.17 mmol) $\mathrm{CuBr}_{2}$ in $10 \mathrm{~mL}$ EtOH erhalten. Ausbeute: $0.04 \mathrm{~g}(42 \%)$. - Schmp.: > $220{ }^{\circ} \mathrm{C}$ (Zers.). $\mathrm{C}_{20} \mathrm{H}_{36} \mathrm{Br}_{2} \mathrm{CuN}_{4}$ (555.88): ber. C 43.21, $\mathrm{H} 6.53, \mathrm{~N} 10.08$; gef. C 42.16, H 6.85, N 10.24.

\section{$\left[\mathrm{TmCl}\left(\mathrm{CH}_{2} \mathrm{CH}_{2} \mathrm{NCH}_{2} \mathrm{CH}=\mathrm{CH}_{2}\right)_{4}\right] \mathrm{Cl}_{2}\left(\mathrm{H}_{2} \mathrm{O}\right)_{6}(8 \mathrm{a})$}

In Analogie zur Darstellung von 3a wird beige farbenes 8a durch Umsetzung von $0.25 \mathrm{~g}(0.75 \mathrm{mmol}) \mathbf{2 a} \mathrm{mit}$ $0.28 \mathrm{~g}(0.85 \mathrm{mmol}) \mathrm{TmCl}_{3}\left(\mathrm{H}_{2} \mathrm{O}\right)_{6}$ in $20 \mathrm{~mL}$ THF nach $48 \mathrm{~h}$ Rühren des Reaktionsgemischs bei $60{ }^{\circ} \mathrm{C}$ und entsprechender Aufarbeitung erhalten. Ausbeute: $0.21 \mathrm{~g} \mathrm{(40 \% ).-}$ Schmp.: $>250{ }^{\circ} \mathrm{C}$ (Zers.). $-\mathrm{C}_{20} \mathrm{H}_{48} \mathrm{Cl}_{3} \mathrm{~N}_{4} \mathrm{O}_{6} \mathrm{Tm}$ (715.91): ber. C 33.55, H 6.76, N 7.85; gef. C 32.67, H 5.45, N 7.10.

\section{$\left[\mathrm{TmCl}\left(\mathrm{CH}_{2} \mathrm{CH}_{2} \mathrm{NCH}_{2} \mathrm{CH}_{2} \mathrm{CH}=\mathrm{CH}_{2}\right)_{4}\right] \mathrm{Cl}_{2}(\boldsymbol{8} \boldsymbol{b})$}

In Analogie zur Darstellung von 3a wird beige farbenes $\mathbf{8 b}$ durch Umsetzung von $0.25 \mathrm{~g}(0.64 \mathrm{mmol}) \mathbf{2 b}$ mit $0.24 \mathrm{~g}(0.75 \mathrm{mmol}) \mathrm{TmCl}_{3}\left(\mathrm{H}_{2} \mathrm{O}\right)_{6}$ in $20 \mathrm{~mL}$ THF nach $24 \mathrm{~h}$ Rühren des Reaktionsgemischs bei $60{ }^{\circ} \mathrm{C}$ und entsprechender Aufarbeitung erhalten. Ausbeute: $0.36 \mathrm{~g}(84 \%)$. $\mathrm{C}_{24} \mathrm{H}_{44} \mathrm{Cl}_{3} \mathrm{~N}_{4} \mathrm{Tm}$ (663.93): ber. C 43.42, H 6.68, N 8.44; gef. C 44.00, H 6.36, N 8.34.

\section{$\left\{\left[\mathrm{NiCl}\left(\mathrm{CH}_{2} \mathrm{CH}_{2} \mathrm{NCH}_{2} \mathrm{CH}=\mathrm{CH}_{2}\right)_{4}\right]_{2}[\mathrm{CuCl}]_{6}\right\}_{n}(\mathbf{9})$}

$\mathrm{Zu}$ einer Lösung von $0.06 \mathrm{~g}(0.59 \mathrm{mmol}) \mathrm{CuCl}$ in $10 \mathrm{~mL}$ $\mathrm{CH}_{3} \mathrm{CN}$ wird bei Raumtemperatur eine Lösung von $0.07 \mathrm{~g}$ ( $0.15 \mathrm{mmol})$ 6a in $10 \mathrm{~mL} \mathrm{CH}_{3} \mathrm{CN}$ getropft. Die Reaktionsmischung wird $24 \mathrm{~h}$ bei Raumtemperatur gerührt, durch Abdestillieren des Lösemittels eingeengt und dann von ungelösten Bestandteilen abfiltriert. Innerhalb von 48 h kristallisiert 9 aus der konzentrierten Lösung in Form dunkelgrüner, rautenförmiger Kristalle. Ausbeute: $0.05 \mathrm{~g}(42 \%)$.

\section{Einkristall-Röntgenstrukturanalyse}

Zur Röntgenstrukturanalyse geeignete Einkristalle von 2a.3HCl konnten aus Wasser, von 7 aus Ethanol/Wasser, von $6 \mathbf{a}$ und 9 aus Acetonitril und von 6b aus Methanol erhalten werden. Sie wurden mit einem Diffraktometer Siemens SMART CCD unter Verwendung von $\mathrm{MoK} \alpha^{-}$ Strahlung (Graphitmonochromator) bei $173 \mathrm{~K}$ untersucht. Die vorläufige Bestimmung der Gitterkonstanten erfolgte aus 45 "Frames" (Schrittweite $0.3^{\circ}$ in $\omega$ ). Die endgültigen Konstanten wurden durch Verfeinerung der Reflexe, die aus der Integration aller Frame-Daten erhalten wurden, ermittelt. Die Messung erfolgte mit einer Schrittweite von $0.3^{\circ}$ in $\omega$, einer Zählzeit von $5 \mathrm{~s}$ pro Frame und einem Kristall- 
Detektor-Abstand von $3 \mathrm{~cm}$ unter Verwendung des Messprogramms SMART [27]. Die Auswertung erfolgte unter Verwendung des Programms SAINT [27]. Es wurden empirische Absorptionskorrekturen (SADABS [28]) durchgeführt. Die Strukturlösung erfolgte mit Direkten Methoden (SHELXS97 [29]), die Verfeinerung mit SHELXTL [30]. Alle Nichtwasserstoffatome wurden im Allgemeinen anisotrop verfeinert. Bei einigen Strukturen wurden die Atome fehlgeordneter Bereiche nur isotrop verfeinert. Wasserstoffatome wurden mit konstanten isotropen Temperaturfaktoren $\left(U_{\text {iso }}=0.08 \AA^{2}\right)$ in idealisierten Positionen berechnet. Das Programm DiAMOND [31] wurde für die Strukturdarstellung verwendet. In Tabelle 1 sind die Kristalldaten und Messparameter zusammengefasst. Aufgrund fehlgeordneter Lösemittelmoleküle in den Kristallen und Fehlordnungen der Alkylenketten in den Molekülen weisen die Strukturbestimmungen teilweise recht schlechte $R$-Werte auf. Die Abstände für die $\mathrm{C}-\mathrm{C}$ - und $\mathrm{C}-\mathrm{N}$-Bindungen liegen jedoch bei al- len Verbindungen im Erwartungsbereich für derartige Bindungen, durch Fehlordnung abweichend erscheinende Bindungslängen werden nicht diskutiert. Die Molekülstrukturen der Verbindungen sind in Abb. 1-5 dargestellt, dabei sind zum Teil Wasserstoffatome und Atome auf Splitpositionen (bei fehlgeordneten Bereichen) der Übersichtlichkeit halber weggelassen. In den Legenden zu den Abb. sind einige wichtige Bindungslängen und -winkel angegeben.

CCDC 710630 (2a), 710631 (6a), 710632 (6b), 710633 (7), bzw. 710634 (9) enthalten die beim Cambridge Crystallographic Data Centre hinterlegten Kristallstrukturdaten. Anforderung: www.ccdc.cam.ac.uk/data_request/cif.

Dank

Wir danken Frau Priv.-Doz. Dr. Petra Escarpa-Gaede für die Aufnahme der Cyclovoltagramme, der Degussa AG für $\mathrm{RhCl}_{3}\left(\mathrm{H}_{2} \mathrm{O}\right)_{6}$ und dem Fonds der Chemischen Industrie für finanzielle Unterstützung dieser Arbeit.
[1] E. Kimura, T. Koike, Chem. Commun. 1998, 1495.

[2] E. Kimura, T. Koike, M. Shionoya, Struct. Bonding 1997, 89, 1.

[3] E. Kimura, T. Koike, Advances in Inorganic Chemistry, Vol. 44, Academic Press, New York, 1996, p. 229.

[4] S. Blain, P. Appriou, H. Chaumeil, H. Handel, Anal. Chim. Acta 1990, 232, 331.

[5] E. Kimura, T. Koike, Chem. Soc. Rev. 1998, 179.

[6] J. C. Bousquet, S. Saini, D. D. Stark, P. F. Hahn, M. Nigam, J. Wittenberg, J. Ferrucci, Radiology 1988, 166, 693.

[7] H. Tsubuke, T. Yoden, T. Iwachido, M. Zenki, J. Chem. Soc., Chem. Commun. 1991, 1069.

[8] H.F. Handel, R. Muller, R. Guglielmetti, Helv. Chim. Acta 1983, 66, 514.

[9] R. A. Bullman, Struct. Bonding 1987, 41.

[10] D. Bryce-Smith, Chem. Soc. Rev. 1986, 15, 93.

[11] Y.H. Jang, M. Blanco, S. Dasgupta, D. A. Keire, J. E. Shively, A. Goddard III, J. Am. Chem. Soc. 1999, 121, 6124.

[12] K. E. Borbas, C. S. M. Ferreira, A. Perkins, J. I. Bruce, S. Missailidis, Bioconjugate Chem. 2007, 18, 1205.

[13] T. Gunnlaugsson, J. P. Leonard, Chem. Commun. 2005, 3114.

[14] K. Michaelis, M. Kalesse, Angew. Chem. 1999, 111, 2832; Angew. Chem. Int. Ed. 1999, 38, 2243.

[15] C. Trabaud, J. Dessolin, P. Vlieghe, M. Bouygues, J. C. Shermann, M. Camplo, J. L. Kraus, Bioorg. Med. Chem. Lett. 1997, 7, 1353.

[16] G. J. Bridger, R.T. Skerlj, S. Padmanabhan, S. A. Martellucci, G. W. Henson, S. Struyf, M. Witvrouw, D. Schols, E. De Clerq, J. Med. Chem. 1999, 42, 3971.

[17] Y. Inoue, E. Kimura, Biol. Pharm. Bull. 1998, 19, 456.

[18] D. J. Kong, L. H. Meng, J. Ding, Y. Y. Xie, X. Y. Huang, Polyhedron 2000, 19, 217.
[19] a) R. M. Izatt, K. Pawlack, J. S. Bradshaw, R. L. Brüning, Chem. Rev. 1995, 95, 2529; b) K. P. Wainwright, Coord. Chem. Rev. 1997, 166, 35; c) M. Meyer, V. Dahaoui-Gindrey, C. Lecomte, R. Guilard, Coord. Chem. Rev. 1998, 180, 1313.

[20] M. V. Baker, D. H. Brown, B. W. Skelton, A. H. White, Aust. J. Chem. 2002, 55, 655.

[21] Spectral Database for Organic Compounds, SDBS; see: http://riodb01.ibase.aist.go.jp/sdbs/cgi-bin/cre_ index.cgi?lang=eng

[22] J. S. Thompson, R. L. Harlow, J.F. Whitney, J. Am. Chem. Soc. 1983, 105, 3522.

[23] T. J. Atkins, J.E. Richman, W. F. Bulkowski, J. Org. Chem. 1978, 58, 86.

[24] J. E. Richman, T. J. Atkins, J. Am. Chem. Soc. 1974, 96, 2268.

[25] D. Parker, Macrocycle Synthesis. A. Practical Approach. Oxford University Press, Oxford, 1996.

[26] S. W. Kohl, K. Kuse, M. Hummert, H. Schumann, C. Mügge, K. Janek, H. Weißhoff, Z. Naturforsch. 2007, 62b, 397.

[27] Smart, Saint, Software Reference Manual (version 4.0), Siemens Analytical X-ray Instruments Inc., Madison, Wisconsin (USA) 1996.

[28] G. M. Sheldrick, SADABS, Program for Empirical Absorption Correction of Area Detector Data, University of Göttingen, Göttingen (Germany) 1996.

[29] G. M. Sheldrick, SHELXS-97, Program for the Solution of Crystal Structures, University of Göttingen, Göttingen (Germany) 1997.

[30] G. M. Sheldrick, SHELXTL, Reference Manual (version 5.1), Bruker AXS, Madison, WI (USA) 1997.

[31] K. Brandenburg, DiAmond, Crystal and Molecular Structure Visualization, Crystal Impact - K. Brandenburg \& H. Putz GbR, Bonn (Germany) 1999. 